Article

\title{
Agreement Between Dribble and Change of Direction Deficits to Assess Directional Asymmetry in Young Elite Football Players
}

\author{
Athos Trecroci ${ }^{1}{ }^{(}$, Tindaro Bongiovanni ${ }^{2}{ }^{(D}$, Luca Cavaggioni ${ }^{1,3}$, Giulio Pasta ${ }^{4}$, \\ Damiano Formenti ${ }^{5, *}$ and Giampietro Alberti ${ }^{1}$ \\ 1 Department of Biomedical Sciences for Health, Università degli Studi di Milano, 20122 Milano, Italy; \\ athostrec@gmail.com (A.T.); cavaggioni.luca@gmail.com (L.C.); giampietro.alberti@unimi.it (G.A.) \\ 2 Nutrition, Hydration \& Body Composition Department, Parma Calcio 1913, 43044 Parma, Italy; \\ tindaro.bongiovanni@gmail.com \\ 3 Department of Endocrine and Metabolic Diseases, 20021 Milan, Italy \\ 4 Medical Department, Parma Calcio 1913, 43044 Parma, Italy; ghitopasta@hotmail.com \\ 5 Department of Biotechnology and Life Sciences (DBSV), University of Insubria, 21100 Varese, Italy \\ * Correspondence: damiano.formenti@uninsubria.it; Tel.: +39-347-4128375
}

Received: 1 April 2020; Accepted: 5 May 2020; Published: 8 May 2020

\begin{abstract}
This study aimed to examine the agreement between asymmetries of dribble and change of direction (COD) deficits and to determine their potential difference to each other. Sixteen young elite football players were recruited and tested for sprint (over $10 \mathrm{~m})$, dribbling $\left(90^{\circ} \mathrm{COD}_{\text {dribbling }}\right.$ ) and $\mathrm{COD}\left(90^{\circ} \mathrm{COD}_{\text {running }}\right)$ performance in dominant (fastest) and non-dominant (slowest) directions. Dribble and COD deficits were computed to express dribbling and COD ability without the influence of acceleration. The asymmetric index (AI\%) of both dribble and COD deficits were obtained for both directions. The level of agreement between dribble and COD deficits was assessed by Cohen's kappa statistic $(\kappa)$. Results showed that AI\% measured by dribble and COD deficits presented a poor level of agreement $(\kappa=-0.159)$, indicating their imbalance did not favor the same direction. Moreover, $\mathrm{AI} \%$ of the dribble deficit was significantly higher than those of the COD deficit. This study demonstrated that asymmetries in dribbling and change of direction performance (measured by dribble and COD deficit) were not in agreement to favor the same direction, also displaying a significant difference to each other. Practitioners should consider the task-specificity of asymmetry to reduce the imbalance in dribbling and COD performance.
\end{abstract}

Keywords: agreement; imbalance; football skills; football performance

\section{Introduction}

The combined asymmetrical and unpredictable nature of football prompts each player dribbling or changing direction in multiple directions (chaotically) within the pitch, which is unlikely to be equally distributed during a match [1]. Moreover, additional inherent factors (e.g., playing position, tactical constraints and players' leg or directional preference) may also contribute to influencing the players' movements within the pitch favoring predominantly their dominant side or direction to the detriment of the non-dominant one [2]. Although it would be advantageous for team sport athletes to express similar dribbling and change of direction (COD) performance toward different directions (right versus left) [3], they often manifest a certain degree of asymmetry, even throughout the season $[1,4]$, that should be opportunely quantified.

Despite the apparent relevance of assessing dribbling and change of direction (COD) asymmetries, the available literature is scarce. Most of the studies used the completion time (the total time to cover a 
specific course) to detect the dribbling [5] and COD performance [2,6] for imbalance purposes. It has been previously observed that completion time might be biased by an individual's sprint capacity either via dribbling $[7,8]$ or changing direction assessment $[9,10]$. To overcome this issue, dribble and COD deficits have been proposed to provide practitioners with a more valid and isolated measure within a field-based context, limiting the impact of acceleration [7-10].

A recent study quantified the directional asymmetry in the 505 COD test by deficit and total time between groups of different team-sport athletes (e.g., football, basketball and cricket). It was found that all athletes manifested a certain degree of asymmetry between the dominant and non-dominant side for both COD deficit and total time [11]. The authors also concluded that, being COD deficit unbiased toward individuals with higher acceleration capacity, its use should be preferred to compare asymmetry in respect of total time [11]. Moreover, in the studies of Dos'Santos et al. [3,12], the asymmetry of COD deficit reported higher percentages compared with total time with $35 \%$ of the subjects exhibiting values greater than the asymmetric threshold (14.5\%) and $49 \%$ showing asymmetries greater than $10 \%$ [3], which has been previously used as a limit for an acceptable imbalance [12-14]. Although a dearth of research exists on COD deficit asymmetries, no information is available on the use of dribble deficit to quantify directional asymmetry.

Following the available literature, different assessments of asymmetry over a certain task may detect diverse levels of imbalances rarely favoring the same side or direction [15]. Madruga et al. [16] investigated whether the asymmetry was consistent between three unilateral jump-based tests in team sport athletes. The authors reported a low level of agreement suggesting that the asymmetries rarely favored the same dominant side. Similarly, Bishop et al. [17] reported slight to a fair agreement in the asymmetry within unilateral strength and jumping-based tests. Taken all together, these findings highlight the task-specificity of asymmetry, which should be considered when interpreting any performance influenced by leg or directional dominance in team sports. This may have important implications on the assessment of dribbling and COD asymmetries in football players. Dribbling and changing direction is pivotal to successfully compete in football. Quick and accurate change of directions while dribbling a ball allows a player to pass her or his opponent more easily, to invade a specific field area and to create a numerical superiority for increasing any chance of scoring a goal. In this context, besides quantifying the asymmetry using dribble and COD deficits, knowing whether there is consistency across them would be of practical importance. Of note, this may provide practitioners with useful information to target additional exercises for each individual's dominant (faster or preferred) and non-dominant (slower or non-preferred) side [17], which might differ between dribbling and CODs.

Therefore, the aim of the study was twofold: i) to examine the degree of agreement between dribble and COD deficit asymmetries in favoring the same direction; ii) to determine the extent of each dribble and COD deficit asymmetry and the possible difference to each other. Dribbling and COD are different movement tasks with the former more complex and technically demanding, especially concerning dominant and non-dominant sides [5]. Given the supposed task-specificity of asymmetry, we hypothesized that asymmetries of dribble and COD deficit would not favor the same direction, with the former displaying greater values than the latter.

\section{Materials and Methods}

\subsection{Experimental Approach}

In this cross-sectional study, 16 young football players from a professional club were tested for their dribbling and change of direction ability via a $90^{\circ} \mathrm{COD}$ test (for both dominant and non-dominant directions) over $10 \mathrm{~m}$ (with 5-m entry and exit). Dribble and COD deficits were employed to offer an actual ability to dribble or change direction without the influence of acceleration capacity. Then, the asymmetry index of dribble and COD deficit was computed to establish their level of agreement 
through the kappa coefficient. The derived asymmetries were also compared to each other to detect whether a potential difference would exist between dribbling and changing direction.

\subsection{Subjects}

Sixteen young elite football players from the same professional club (age $14.5 \pm 0.8$ years, body weight $64.3 \pm 6.2 \mathrm{~kg}$, height $177.1 \pm 4.9 \mathrm{~cm}$, maturity offset $1.05 \pm 0.30$ years) voluntarily participated in the study. The selected sample size was above the minimum value requested for conducting a Cohen's kappa agreement study [18]. All participants and their parents or guardians were informed about the purpose and potential experimental risks. After a deep description of the study, written consent was obtained from subjects and their parents or guardians to participate in the investigation. The study was approved by the Ethical Committee of the local Institution, in accordance with the Helsinki's declaration.

\subsection{Testing Procedures}

The subjects took part in the experimental procedure in June and were tested on an outdoor artificial turf at the same time of the day (i.e., from 3 p.m. to 5 p.m.). The subjects participated in two sessions. The first session involved a familiarization procedure in which all subjects gained confidence with the testing battery. Additionally, height, sitting height and body mass were taken by a stadiometer (SECA 213, Germany) and a portable scale (813, Germany) to the nearest of $1.0 \mathrm{~cm}$ and $0.1 \mathrm{~kg}$, respectively. In the second session, a testing battery including 10-m sprint and $90^{\circ} \mathrm{COD}$ test (executed with and without a ball) was randomly arranged. A 5-min standardized warm-up based on forward and backward jogging, acceleration, deceleration and skipping movements up to $5 \mathrm{~m}$, was employed before undertaking the first test [19]. An electronic timing gates system (Witty, Microgate, Bolzano, Italia) was used to record the total time for 10-m sprint, dribbling and COD performance with the gates set at $0.7 \mathrm{~m}$ above the ground. The foremost foot was placed $0.3 \mathrm{~m}$ behind the starting line.

\subsubsection{Sprint Assessment}

Each subject, when ready, sprinted over a $10 \mathrm{~m}$ from a two-point staggered stance. The subjects performed three maximal efforts interspersed by 2 minutes of passive recovery. The best performance time was considered in the analysis.

\subsubsection{Dribbling and Change of Direction Assessment}

A $90^{\circ}$ change of direction test for dribbling $\left(90^{\circ} \mathrm{COD}_{\text {dribbling }}\right)$ and running $\left(90^{\circ} \mathrm{COD}_{\text {running }}\right)$ was employed. The layout of the test is shown in Figure 1. All players were instructed to perform three bouts with the ball and three bouts without the ball for each direction (right and left) with 2 minutes of passive recovery in between. The best performance of the three bouts (in each direction) was considered for subsequent analysis. The distance between the starting line to the cone and between

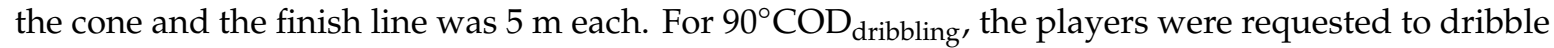
the ball around the cone with a minimum of two touches (with the same foot) along each 5-m path. For $90^{\circ} \mathrm{COD}_{\text {running, }}$, they were instructed to change direction around the cone using the same side-step technique in each bout, to avoid any influence due to different COD execution technique. In case of hitting or touching the cone (even with the ball) at the turning point, the player was stopped and invited to repeat the bout after 2 minutes of recovery. The $90^{\circ} \mathrm{COD}_{\text {dribbling }}$ and $90^{\circ} \mathrm{COD}_{\text {running }}$ performance were initially measured by the total running time to complete the $5-\mathrm{m}+5-\mathrm{m}$ course. Based on the recommendations of previous studies [3,9], we decided to employ a COD deficit for inferential analysis on asymmetry while using total running time for descriptive purposes. The dribble deficit was calculated by subtracting the $90^{\circ} \mathrm{COD}_{\text {running }}$ total time from the $90^{\circ} \mathrm{COD}_{\text {dribbling }}$ total time. The COD deficit was calculated by subtracting the $10-\mathrm{m}$ sprint time from the $90^{\circ} \mathrm{COD}_{\text {running }}$ total time. The fastest mean value between right and left directions was deemed as dominant (D) and the slowest mean value was considered as non-dominant (ND) [3]. 


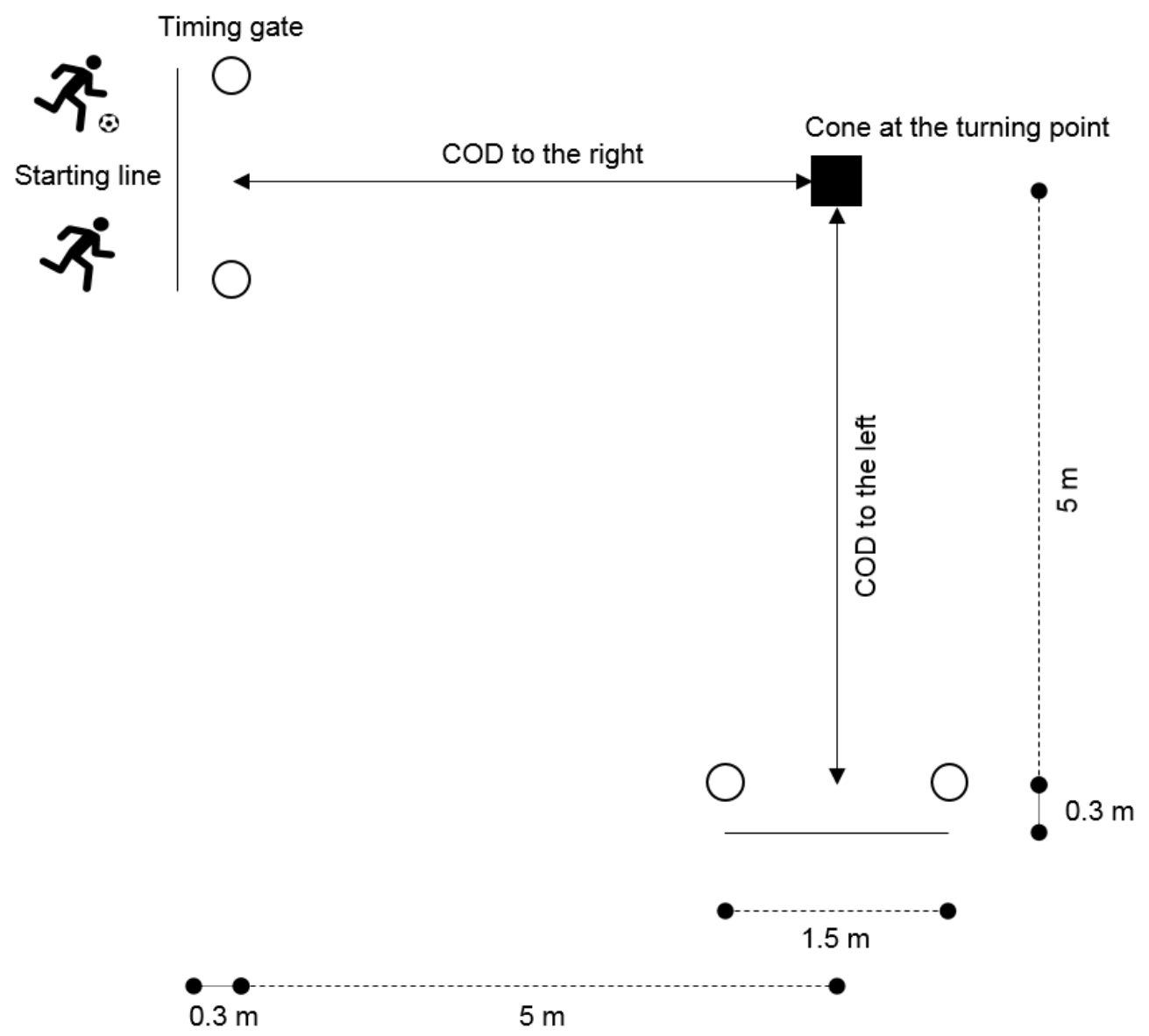

Figure 1. The layout of the $90^{\circ}$ change of direction (COD) test. The black silhouettes with and without the ball identify the dribbling and COD performance, respectively.

\subsubsection{Asymmetric Index Calculation}

For both dribble and COD deficits the asymmetry index (AI\%) was computed with the following formula:

$$
\mathrm{AI} \%=((\mathrm{D}-\mathrm{ND}) / \mathrm{D}) \times 100
$$

Likewise, as previously proposed by Dos'Santos et al. [3], an asymmetry threshold (AT\%) was also obtained to determine whether an individual can be considered as asymmetrical with the formula [3]:

$$
\mathrm{AT} \%=\mathrm{AI} \% \text { mean }+(0.2 \times \mathrm{SD})
$$

where $\mathrm{SD}$ is the standard deviation of the $\mathrm{AI} \%$ mean.

\subsection{Statistical Analysis}

The Shapiro-Wilk's test was conducted to verify if all data were normally distributed. The AI\% resulted in non-normal distribution. Relative and absolute reliability was assessed for all tests using the intra-class correlation coefficient (ICC), standard error of the measurement (SEM) and the coefficient of variation (CV), respectively. Paired t-tests or Wilcoxon signed-rank test were used to detect differences between $\mathrm{D}$ and ND directions in $90^{\circ} \mathrm{COD}_{\text {running }}$ (for total running time and COD deficit) and $90^{\circ} \mathrm{COD}_{\text {dribbling }}$ (for total dribbling time and dribble deficit) tests, and between the $\mathrm{AI} \%$ of dribble and COD deficit, respectively. The effect size of each difference was detected by Cohen's $d(d)$ computation. The corresponding $d$ was classified as trivial $(d<0.2)$, small $(0.2<d<0.6),(0.6<d<1.2)$ moderate, $(1.2<d<2.0)$ large, $(2.0<d<4.0)$ very large and $(d>0.8)$ near perfect. 
The degree of agreement between the AI\% of dribble and COD deficit was assessed by Cohen's kappa statistic $(\kappa)$. We used $\kappa$ coefficient as an appropriate tool for assessing the agreement of directional asymmetry (between the two tests) involving right and left dichotomous variables. The $\kappa$ coefficient described the chance-corrected proportional agreement determining how consistently an asymmetry in dribble and COD deficit agreed on the same direction $[20,21]$. Specifically, $\kappa$ was given by the formula:

$$
\kappa=(\text { Observed Agreement }- \text { Chance agreement }) /(\text { Maximum agreement }- \text { Chance agreement })
$$

where the observed agreement defines the percentage proportion of the directions (right and left) for which dribble and COD deficit agree, and the chance agreement defines the overall random agreement probability that they agree on the same direction. According to Viera and Garrett [20], the following levels of agreement were considered: $\kappa<0.00$ (poor), $0.01<\kappa<0.20$ (slight), $0.21<\kappa<0.400$ (fair), $0.41<\kappa<0.60$ (moderate), $0.61<\kappa<0.80$ (substantial) and $0.81<\kappa<0.99$ (almost perfect). Statistical analysis was performed using IBM Statistical Package for the Social Science version 21.0 (IBM Corp.; Armonk, NY, USA). An $\alpha$-value of 0.05 was set as a criterion level of significance. Ninety-five percent confidence intervals $(95 \% \mathrm{CI})$ are shown in squared brackets. Data are reported as mean \pm standard deviation (SD).

\section{Results}

ICC values showed excellent reliability in $10 \mathrm{~m}$ sprint (ICC $=0.95,95 \%$ CI [ 0.86 to 0.98 ]; $S E M=0.02 \mathrm{~s}$, $\mathrm{CV}=1.8 \%), 90^{\circ} \mathrm{COD}_{\text {running }}$ test for $\mathrm{D}(\mathrm{ICC}=0.93,95 \% \mathrm{CI}[0.88-0.96] ; \mathrm{SEM}=0.03, \mathrm{CV}=2.3 \%$ ) and ND directions (ICC $=0.94,95 \% \mathrm{CI}[0.86-0.95]$; $\mathrm{SEM}=0.03, \mathrm{CV}=2.5 \%), 90^{\circ} \mathrm{COD}_{\text {dribbling }}$ test for $\mathrm{D}$ (ICC $=0.88,95 \% \mathrm{CI}[0.61$ to 0.96$] ; \mathrm{SEM}=0.097 \mathrm{~s}, \mathrm{CV}=3.3 \%$ ) and ND directions (ICC $=0.88,95 \% \mathrm{CI}$ [0.66 to 0.96]; $\mathrm{SEM}=0.105, \mathrm{CV}=3.5 \%$ ). The descriptive statistics of each performance outcome with the inclusion of asymmetry are shown in Table 1 . In $90^{\circ} \mathrm{COD}_{\text {running }}$ test, significant differences were observed between D and ND for total running time and COD deficit with large and moderate effects, respectively ( $\mathrm{p}<0.0001, d=-1.07,95 \% \mathrm{CI}[-1.84$ to -0.30$]$ and $p<0.0001, d=-0.73,95 \% \mathrm{CI}[-1.47$ to 0.00], respectively). Likewise, in $90^{\circ} \mathrm{COD}_{\text {dribbling }}$ test, significant differences were observed between $\mathrm{D}$ and ND for total dribbling time and dribble deficit with small effects $(p<0.0001, d=-0.55,95 \%$ CI [ -1.28 to 0.17$]$ and $p<0.0001, d=-0.57,95 \%$ CI [-1.30 to 0.15$]$, respectively). The Wilcoxon signed-rank test revealed a significant difference between $\mathrm{AI} \%$ of dribble deficit and $\mathrm{AI} \%$ of $\mathrm{COD}$ deficit $(\mathrm{Z}=-2.275$, $p=0.021$ ). The AT $\%$ of dribble and COD deficits were $17.22 \%$ and $41.62 \%$, respectively.

Table 1. Descriptive statistics of performance outcomes.

\begin{tabular}{|c|c|c|}
\hline Physical Performance Tests & Mean \pm SD & $95 \%$ CI \\
\hline \multicolumn{3}{|l|}{$10 \mathrm{~m}$ sprint } \\
\hline Sprint running time (s) & $1.89 \pm 0.09$ & 1.83 to 1.94 \\
\hline \multicolumn{3}{|l|}{$90^{\circ} \mathrm{COD}$ running } \\
\hline Total running time $\mathrm{D}^{* * * a}(\mathrm{~s})$ & $2.43 \pm 0.06$ & 2.39 to 2.46 \\
\hline Total running time ND (s) & $2.50 \pm 0.07$ & 2.46 to 2.54 \\
\hline COD deficit $\mathrm{D}^{* * * \mathrm{~b}}(\mathrm{~s})$ & $0.54 \pm 0.09$ & 0.49 to 0.59 \\
\hline COD deficit ND (s) & $0.61 \pm 0.10$ & 0.56 to 0.67 \\
\hline \multicolumn{3}{|l|}{$90^{\circ} \mathrm{COD}$ dribbling } \\
\hline Total dribbling time $\mathrm{D}^{* * * \mathrm{c}}(\mathrm{s})$ & $2.84 \pm 0.14$ & 2.76 to 2.92 \\
\hline Total dribbling time ND (s) & $2.93 \pm 0.18$ & 2.84 to 3.03 \\
\hline Dribble deficit $D^{* * * c}(\mathrm{~s})$ & $0.33 \pm 0.10$ & 0.27 to 0.38 \\
\hline Dribble deficit ND (s) & $0.43 \pm 0.12$ & 0.37 to 0.50 \\
\hline \multicolumn{3}{|l|}{ Asymmetry } \\
\hline AI COD deficit (\%) & $-14.62 \pm 13.03$ & -21.56 to -7.67 \\
\hline AI Dribble deficit * $(\%)$ & $-36.11 \pm 27.56$ & -50.80 to -21.42 \\
\hline
\end{tabular}

*** Significant $(p<0.0001)$ difference from ND, * Significant $(p<0.05)$ difference from AI COD deficit $(\%) .{ }^{a}$ Large effect size $d$ versus ND, ${ }^{\mathrm{b}}$ moderate effect size $d$ versus ND, ${ }^{\mathrm{s}}$ small effect size $d$ versus ND. Note: $\mathrm{D}=$ dominant, $\mathrm{ND}=$ non-dominant, $\mathrm{COD}=$ change of direction speed, $\mathrm{AI}=$ asymmetry index, $\mathrm{SD}=$ standard deviation, $\mathrm{CI}=$ confidence interval. 
Figure 2 shows the individual data for dribble and COD deficit asymmetries. In 6 out of 16 players, the two AIs $\%$ favored the same direction with a resultant observed agreement of $0.38(38 \%)$. The random probability agreement that dribble and COD deficit favored the right and left directions were $~ 39 \%$ and $\sim 10 \%$, respectively, with a chance-corrected proportional agreement of $0.46(46 \%)$. The resultant $\mathrm{k}$ score indicated a poor agreement of -0.159 (standard error $=0.187,95 \% \mathrm{CI}[-0.526$ to 0.208$]$ ) between AIs\% of dribble and COD deficits in favoring the same direction.

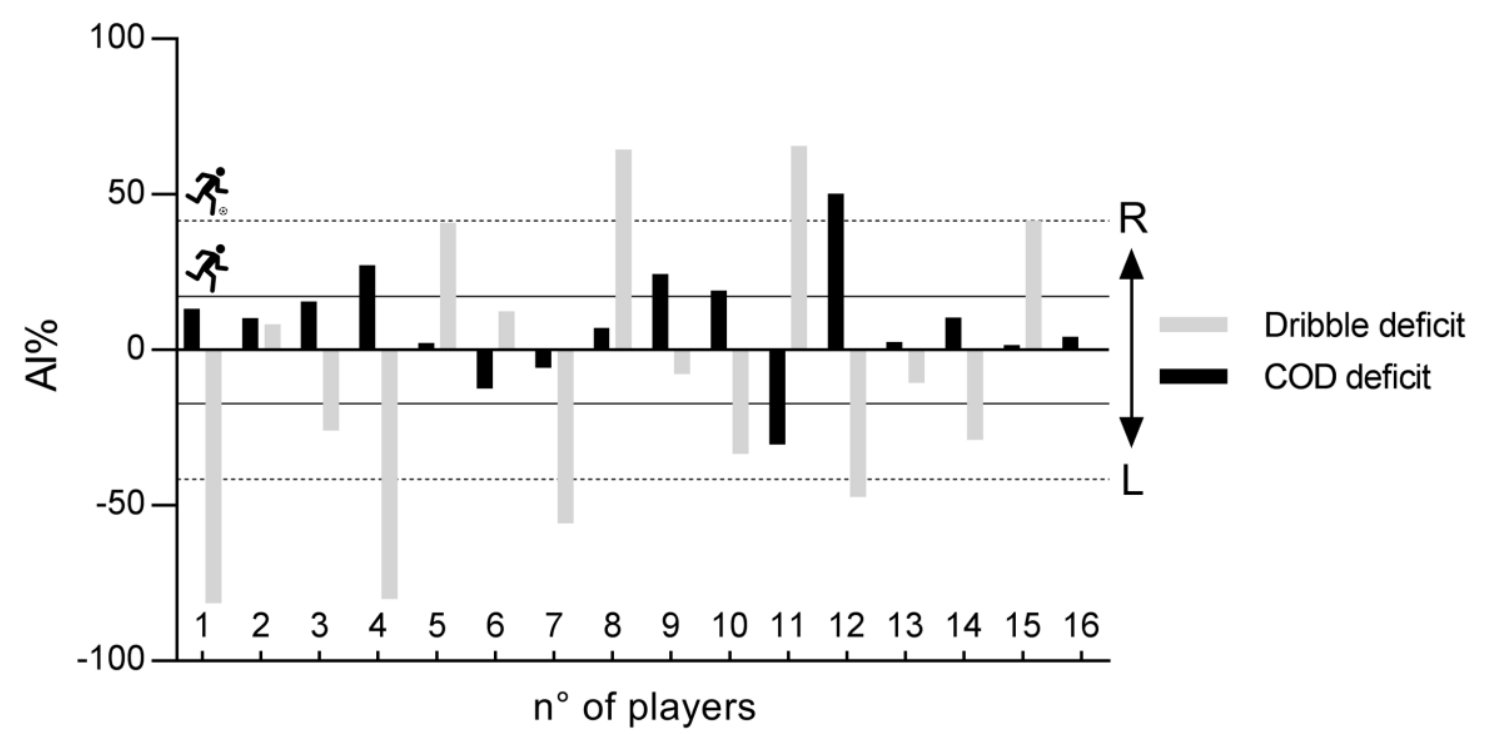

Figure 2. Individual asymmetry data (AI\%) for dribble and change of direction (COD) deficits. Bars above the 0 line shows the asymmetry favoring the right direction, and bars below the 0 line shows the asymmetry favoring the left direction. The dotted lines indicate the asymmetry threshold of dribble deficit while the solid lines indicate the asymmetry threshold of COD deficit, respectively.

\section{Discussion}

The main finding of this study was that the asymmetry measured by dribble and COD deficits presented a poor level of agreement, indicating they did not favor the same direction. Moreover, it has been shown that, on average, AIs\% of dribble and COD deficits were significantly different from each other, with the former presenting the highest values. These findings are in line with our hypothesis that dribble and COD deficits would not exhibit asymmetries favoring the same direction, with the former displaying the highest value.

Demonstrating whether (or not) the level of asymmetry (i.e., right versus left) is consistent across dribbling and COD performance provides practitioners with practical information that can be helpful to design targeted training strategies. According to the present results, while a player exhibited a fast change direction with the ball on a given side (right), she or he tended to display a fast change direction without the ball toward an opposite one (left). For example, Figure 2 shows only 6 out of $16(\sim 38 \%)$ players presenting an $\mathrm{AI} \%$ favoring the same side, whereas most of the AIs\% were not consistent across dribble and COD deficits. Indeed, the probability that asymmetries of both dribble and COD deficits would favor the same direction by chance was $46 \%$, which is higher than the observed agreement. As such, the resultant level of $\kappa$ score, which has the peculiarity of removing any agreement by chance, indicated that they did not produce similar results on a given side or direction. Taken all together, these findings also suggest that the asymmetry for $90^{\circ} \mathrm{COD}_{\text {dribbling }}$ and $90^{\circ} \mathrm{COD}_{\text {running }}$ tests over young subjects and using a common metric (e.g., deficit) is task-specific. This is supported by the study of Bishop et al. [17] in which the authors examined whether asymmetries were consistent across unilateral strength and common jumping-based tests (e.g., single-leg countermovement jump and single-leg broad jump) for peak force and impulse (eccentric and concentric). Most of the agreement for peak force $(\kappa=0.05)$ and impulse $(-0.25<\kappa<0.32)$ ranged from slight to fair even across common tests, except 
for the substantial $(\kappa=0.79)$ agreement between single-leg countermovement jump and single-leg broad jump tests for concentric impulse. This provides evidence for the notion that asymmetry is task-specific. In fact, given the current results, practitioners should consider the task-specificity of asymmetry when interpreting dribbling and change of direction performance to implement targeted training strategies for an individual's dominant (faster or preferred) and non-dominant (slower or non-preferred) side [17]. For instance, it has been demonstrated that practicing with an emphasis on the non-preferred side (e.g., ND direction) by increasing accuracy and force in the kicks, ball control and speed may be a good practice to reduce asymmetry in dribbling [5]. Of note, the nature of these two motor actions (dribbling and COD) presents some peculiarities that differ from each other. Indeed, compared with COD, dribbling fast in multiple directions requires players a high technical (bilateral) proficiency to maintain the ball under control, which in turn slows their performance time.

To our knowledge, the present study is the first quantifying and comparing the dribbling and COD performance in young elite football players. Dribbling and COD are pivotal to successfully compete in youth football [22-24] with the former considered a field-based predictor of a player's success in one-to-one duels [25]. Unfortunately, some evidence exists on COD deficit in the current literature $[3,9,26-28]$, there is a dearth of information on dribble deficit $[7,8]$ and no data are available on its asymmetry. The unpredictability of game scenarios, together with inherent factors such as playing position, tactical constraints and players' leg or directional preference, may prompt players choosing predominantly their dominant side (at the expense of the non-dominant one) to address any football-specific maneuver [5]. The current results showed that the mean $\mathrm{AI} \%$ of dribble deficit roughly doubled that of COD. The use of the ball requires players being able to perform complex movements depending on additional factors [29] (e.g., force, accuracy and precision kicking of dominant and non-dominant legs) that are likely to enhance the expected directional asymmetry among individuals [5]. Of note, in Figure 2, $6(\sim 38 \%)$ and $5(\sim 31 \%)$ out of 16 players were asymmetrical, presenting an $\mathrm{AI} \%$ higher than the corresponding AT\% for dribble and COD deficits, respectively. It is worth noticing that their values exceeded the common threshold of $10 \%$, which has been previously used as a limit for an acceptable bilateral imbalance [12-14].

As regards COD deficit asymmetries, the present results appear in line with the study of Dos'Santos et al. [3], in which $35 \%$ of the subjects reported a significantly higher bilateral imbalance in COD deficit (by the 505 COD test) than the corresponding asymmetry threshold. However, according to recent studies, the relevance of any discussion about the thresholds and their capability to detect asymmetry has been questioned [4,15]. Bishop et al. [4,17] reported individual data for unilateral strength and jumping-based tests showing that asymmetries can sometimes be as large as $20 \%-40 \%$ with no bearing on a performance outcome (e.g., during CODs) [4]. Additionally, interpreting mean data without devoting attention to an individual approach would not depict a clear portrait of a player's asymmetry, and her or his training needs to reduce it. The current results appear to be in line with such consideration. Regarding dribble deficit, some individuals (e.g., player $\mathrm{n}^{\circ} 1$ in Figure 2) exhibited an imbalance higher than $50 \%$, which is one and a half times larger than mean SD, while others were about on average (e.g., player $\mathrm{n}^{\circ} 6$ in Figure 2). Thus, it is evident how designing targeted training programs to the player $\mathrm{n}^{\circ} 6$ (with an $\mathrm{AI} \%$ barely below the mean) would not likely contemplate the required additional exercises for the player $n^{\circ} 1$ in an attempt to reduce the highest asymmetry. This information can be of practical relevance as practitioners are helped to plan any additional exercise on a more individual level to reduce asymmetry [17] in both dribbling and change of direction ability. It is notable that while COD asymmetries are detectable among team-sport athletes [11], within a homogeneous group as the present elite players, the inter-variability of dribbling and COD tests would limit the interpretation of the mean values. Bishop et al. [17] suggested to report and compare asymmetries to testing variability (e.g., CV\%). In support of this, the inter-individual variability should be taken into account when attempting to screening young football players [30]. As such, it can be provided relevant information underpinning the monitoring and development of individualized or small-groups program routines [30]. 
This study presents limitations that should be acknowledged. The $90^{\circ} \mathrm{COD}$ test currently selected may be limited to represent the variety of dribbling techniques (close dribbling skills or a combination of long kicks and fast acceleration to run past an opponent) performed in matches [25]. Taking into account the task-specificity of asymmetry, further studies are warranted to examine how the evidence of no agreement with COD would be confirmed within a wider spectrum of dribbling skills. We also put in evidence that our findings cannot be surely extended also to other team sports. For instance, dribbling and COD abilities are determinants component in basketball. Thus, further studies are warranted to examine whether the current disagreement between dribble and COD deficit asymmetries in football would be found also in basketball players who dribble with upper limbs instead of lower limbs. Finally, we put in evidence that the present findings should be interpreted according to maturity status. Indeed, although the current players' maturity offset was fairly homogeneous, it might be possible that different results would come from heterogeneous maturity-related profiles, and consequently leading to different dribbling and COD deficit results.

\section{Conclusions}

This study demonstrated that asymmetries in dribbling and change of direction were not in agreement to favor the same direction, probably reflecting the different nature of these motor actions. As such, practitioners should consider the task-specificity of asymmetry to reduce the imbalance between dominant and non-dominant directions. For example, additional dribbling exercises placing the emphasis on the ND direction may represent a good strategy to improve the ND itself, without affecting the D direction. Of note, practitioners are encouraged to interpret asymmetry data with an individual approach to contemplate the required additional exercises for a given player to reduce her or his imbalance on a more individual level. In young elite players, assessing the direction of asymmetry during dribbling and changing direction appears pivotal to guarantee informative data on their potential individual imbalance. Finally, coaches and practitioners may benefit from data on players' directional imbalances to ameliorate both individual monitoring and training processes across the youth athletic development.

Author Contributions: Conceptualization, A.T. and D.F.; methodology, A.T., L.C. and T.B.; formal analysis, A.T. and D.F.; investigation, A.T., L.C., T.B., G.P. and G.A.; data curation, A.T.; writing-original draft preparation, A.T. and D.F.; writing-review and editing, A.T., T.B., L.C., G.P., D.F. and G.A.; supervision, G.A.; All authors have read and agreed to the published version of the manuscript.

Funding: This research received no external funding.

Conflicts of Interest: The authors declare no conflict of interest.

\section{References}

1. Bishop, C.; Read, P.; Chavda, S.; Jarvis, P.; Brazier, J.; Bromley, T.; Turner, A. Magnitude or direction? seasonal variation of interlimb asymmetry in elite academy soccer players. J. Strength Cond. Res. 2020, 1. [CrossRef] [PubMed]

2. Rouissi, M.; Chtara, M.; Berriri, A.; Owen, A.; Chamari, K. Asymmetry of the modified Illinois change of direction test impacts young elite soccer players' performance. Asian J. Sports Med. 2016, 7, e33598. [CrossRef]

3. Dos'Santos, T.; Thomas, C.; Jones, P.A.; Comfort, P. Assessing asymmetries in change of direction speed performance: Application of change of direction deficit. J. Strength Cond. Res. 2019, 33, 2953-2961. [CrossRef]

4. Bishop, C.; Read, P.; Bromley, T.; Brazier, J.J.; Jarvis, P.; Chavda, S.; Turner, A. The association between interlimb asymmetry and athletic performance tasks: A season-long study in elite academy soccer players. J. Strength Cond. Res. 2020. [CrossRef] [PubMed]

5. Teixeira, L.A.; Silva, M.V.; Carvalho, M. Reduction of lateral asymmetries in dribbling: The role of bilateral practice. Laterality 2003, 8, 53-65. [CrossRef] [PubMed]

6. Hart, N.H.; Spiteri, T.; Lockie, R.G.; Nimphius, S.; Newton, R.U. Detecting deficits in change of direction performance using the preplanned multidirectional australian football league agility test. J. Strength Cond. Res. 2014, 28, 3552-3556. [CrossRef] [PubMed] 
7. Ramirez-Campillo, R.; Gentil, P.; Moran, J.; Dalbo, V.J.; Scanlan, A.T. Dribble deficit enables measurement of dribbling speed independent of sprinting speed in collegiate, male, basketball players. J. Strength Cond. Res. 2019, 1. [CrossRef]

8. Scanlan, A.; Wen, N.; Spiteri, T.; Milanović, Z.; Conte, D.; Guy, J.H.; Delextrat, A.; Dalbo, V.J. Dribble deficit: A novel method to measure dribbling speed independent of sprinting speed in basketball players. J. Sports Sci. 2018, 36, 2596-2602. [CrossRef]

9. Nimphius, S.; Callaghan, S.J.; Spiteri, T.; Lockie, R.G. Change of direction deficit: A more isolated measure of change of direction performance than total 505 time. J. Strength Cond. Res. 2016, 30, 3024-3032. [CrossRef]

10. Nimphius, S.; Callaghan, S.J.; Bezodis, N.E.; Lockie, R.G. Change of direction and agility tests: Challenging our current measures of performance. Strength Cond. J. 2017, 40, 26-38. [CrossRef]

11. Dos'Santos, T.; Thomas, C.; Comfort, P.; Jones, P.A. Comparison of change of direction speed performance and asymmetries between team-sport athletes: Application of change of direction deficit. Sports 2018, 6, 174.

12. Dos'Santos, T.; Thomas, C.; Jones, P.A.; Comfort, P. Asymmetries in single and triple hop are not detrimental to change of direction speed. J. Trainol. 2017, 6, 35-41. [CrossRef]

13. Bishop, C.; Turner, A.; Maloney, S.; Lake, J.; Loturco, I.; Bromley, T.; Read, P. Drop jump asymmetry is associated with reduced sprint and change-of-direction speed performance in adult female soccer players. Sports 2019, 7, 29. [CrossRef] [PubMed]

14. Trecroci, A.; Formenti, D.; Ludwig, N.; Gargano, M.; Bosio, A.; Rampinini, E.; Alberti, G. Bilateral asymmetry of skin temperature is not related to bilateral asymmetry of crank torque during an incremental cycling exercise to exhaustion. PeerJ 2018, 6, e4438. [CrossRef]

15. Bishop, C.; Turner, A.; Jarvis, P.; Chavda, S.; Read, P. Considerations for selecting field-based strength and power fitness tests to measure asymmetries. J. Strength Cond. Res. 2017, 31, 2635-2644. [CrossRef]

16. Madruga-Parera, M.; Bishop, C.; Read, P.; Lake, J.; Brazier, J.; Romero-Rodriguez, D. Jumping-based asymmetries are negatively associated with jump, change of direction, and repeated sprint performance, but not linear speed, in adolescent handball athletes. J. Hum. Kinet. 2020, 71, 47-58. [CrossRef]

17. Bishop, C.; Read, P.; Lake, J.; Chavda, S.; Turner, A. Inter-Limb asymmetries: Understanding how to calculate differences from bilateral and unilateral tests. Strength Cond. J. 2018, 40, 1-6. [CrossRef]

18. Bujang, M.A.; Baharum, N. Guidelines of the minimum sample size requirements for Kappa agreement test. Epidemiol. Biostat. Public Health 2017, 14. [CrossRef]

19. Trecroci, A.; Cavaggioni, L.; Lastella, C.; Broggi, M.; Perri, E.; Iaia, F.M.; Alberti, G. Effects of traditional balance and slackline training on physical performance and perceived enjoyment in young soccer players. Res. Sports Med. 2018, 26, 450-461. [CrossRef]

20. Viera, A.J.; Garrett, J.M. Understanding interobserver agreement: The kappa statistic. Fam. Med. 2005, 37, 360-363.

21. Watson, P.F.; Petrie, A. Method agreement analysis: A review of correct methodology. Theriogenology 2010, 73, 1167-1179. [CrossRef] [PubMed]

22. Trecroci, A.; Longo, S.; Perri, E.; Iaia, F.M.; Alberti, G. Field-based physical performance of elite and sub-elite middle-adolescent soccer players. Res. Sports Med. 2019, 27, 60-71. [CrossRef] [PubMed]

23. Trecroci, A.; Milanović, Z.; Frontini, M.; Marcello, I.F. Physical performance comparison between under 15 elite and sub-elite soccer players. J. Hum. Kinet. 2018, 61, 209-216. [CrossRef] [PubMed]

24. Valente-dos-Santos, J.; Coelho-e-Silva, M.; Duarte, J.; Pereira, J.; Rebelo-Gonçalves, R.; Figueiredo, A.; Mazzuco, M.; Sherar, L.; Elferink-Gemser, M.; Malina, R. Allometric multilevel modelling of agility and dribbling speed by skeletal age and playing position in youth soccer players. Int. J. Sports Med. 2014, 35, 762-771. [CrossRef] [PubMed]

25. Wilson, R.S.; Smith, N.M.A.; de Ramos, S.P.; Giuliano Caetano, F.; Aparecido Rinaldo, M.; Santiago, P.R.P.; Cunha, S.A.; Moura, F.A. Dribbling speed along curved paths predicts attacking performance in match-realistic one vs. one soccer games. J. Sports Sci. 2019, 37, 1072-1079. [CrossRef]

26. Loturco, I.; Nimphius, S.; Kobal, R.; Bottino, A.; Zanetti, V.; Pereira, L.A.; Jeffreys, I. Change-of direction deficit in elite young soccer players: The limited relationship between conventional speed and power measures and change-of-direction performance. Ger. J. Exerc. Sport Res. 2018, 48, 228-234. [CrossRef]

27. Nimphius, S.; Geib, G.; Spiteri, T.; Carlisle, D. "Change of direction" deficit measurement in division I American football players. J. Aust. Strength Cond. 2013, 21, 115-117. 
28. Taylor, J.M.; Cunningham, L.; Hood, P.; Thorne, B.; Irvin, G.; Weston, M. The reliability of a modified 505 test and change-of-direction deficit time in elite youth football players. Sci. Med. Footb. 2019, 3, 157-162. [CrossRef]

29. Duarte, J.P.; Tavares, Ó.; Valente-dos-Santos, J.; Severino, V.; Ahmed, A.; Rebelo-Gonçalves, R.; Pereira, J.R.; Vaz, V.; Póvoas, S.; Seabra, A.; et al. Repeated dribbling ability in young soccer players: Reproducibility and variation by the competitive level. J. Hum. Kinet. 2016, 53, 155-166. [CrossRef]

30. Nikolaidis, P.; Ziv, G.; Lidor, R.; Arnon, M. Inter-individual Variability in Soccer Players of Different Age Groups Playing Different Positions. J. Hum. Kinet. 2014, 49, 213-225. [CrossRef]

(C) 2020 by the authors. Licensee MDPI, Basel, Switzerland. This article is an open access article distributed under the terms and conditions of the Creative Commons Attribution (CC BY) license (http://creativecommons.org/licenses/by/4.0/). 